\title{
THE PACKING AND COVERING OF THE COMPLETE GRAPH I: THE FORESTS OF ORDER FIVE
}

\author{
Y. RODITTY \\ School of Mathematical Sciences \\ Tel-Aviv University, Tel-Aviv, Israel \\ (Received September 15, 1984)
}

ABSTRACT. The maximum number of pairwise edge disjoint forests of order five in the complete graph $K_{n}$, and the minimum number of forests of order five whose union is $k_{n}$, are determined.

KEY WORDS AND PHRASES. Packing, Covering, Decomposition.

1980 MATHEMATICS SUBJECT CLASSIFICATION CODE. $05 C 05$.

\section{INTRCDUCTION}

Graphs in this paper are finite with no multiple edges or loops. Beineke [1] defined the general covering (respectively, packing) problem as follows:

For a given graph $G$ find the minimum (maximum) number of edge disjoint subgraphs of $G$ such that each subgraph has a specified property $P$ and the union of the subgraphs is $G$.

Solutions of these problems are known only for a few properties $P$, when $G$ is arbitrary. In most cases $G$ is taken to be the complete graph $K_{r}$ or the complete bipartite graph $K_{m, n}$ (for particular references one may look at Roditty [2]).

DEFINITION: The complete graph $K_{n}$ is said to have a G-decomposition if it is the union of edge disjoint subgraphs each isomorphic to $G$. We denote such a decomposition by $G \mid K_{n}$.

The G-decomposition problem is to determine the set $N(G)$ of natural numbers such that $K_{n}$ has a G-decomposition if and only if $n \in N(G)$. Note that G-decomposition is actually an exact packing and covering. In the proof of our problems of packing and covering, we make great use of the results obtained for the G-decomposition problem in cases when $G$ has five vertices. As usual $[x]$ will denote the largest integer not exceeding $x$ and $\{x\}$ the least integer not less than $x$. We will let $e(G)$ denote the number of edges of the graph $G$ and $H=\bigcup_{i=1}^{t} G_{i}$ will show that the graph $H$ is the union of $t$ edce disjoint graphs $G_{i}$,
$i=1,2, \ldots, t$.

The Theorem of this paper solves variations of the covering and packing problems for the four graphs below:
(i)
$F_{1}: \begin{array}{rrrrr}x & y & z & u & v \\ 0 & 0 & 0 & 0 & 0\end{array}$
denoted $[(x, y, z)(u, v)]$
$F_{2}: \begin{array}{lllll}x & y & z & u & v \\ 0 & 0 & 0 & 0 & 0\end{array}$
denoted $[(x, y, z, u, v)]$ 
(iii) $F_{3}:$

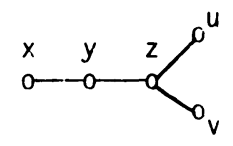

denoted $(x, y, z ; u, v)$

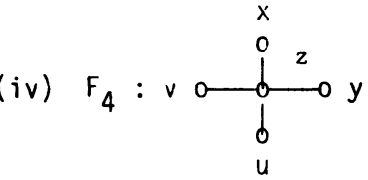

denoted $(z ; x, y, u, v)$

Cur theorem may now be states as THEOREM (Packing and Covering).

let $F$ be $F_{1}, F_{2}$ or $F_{3}$ and $n \geq 5$ or $F$ be $F_{4}$ and $n \geq 7$ then

(i) The maximum number of edge disjoint graphs $F$ which are subgraphs of the complete graph $K_{n}$ is

$$
\left[e\left(K_{n}\right) / e(F)\right]
$$

(ii) The minimum number of graphs $F$ whose union is the complete graph $k_{n}$ is

$$
\left\{e\left(K_{n}\right) / e(F)\right\} .
$$

2. PROOF OF THE THEOREM

We give a separate proof for each choice of $F$.

$F_{1}$ : Froving the Theorem true for $n \geq 5$ is a straightforward exercise. Bermond et a). [3] show that

$$
N\left(F_{1}\right)=\{n \mid n \equiv 0,1(\bmod 3), n \geq 6\}
$$

Thus we have to consider only $n=3 m+2, m \geq 2$. Observe that

$$
K_{3 m+2}=K_{3 m} \cup K_{2,3 m} \cup k_{2}, m \geq 2 .
$$

by (2.1) $K_{3 m}$ has an $F_{1}$-decomposition. Since $K_{2,3 m}=m K_{2,3}$ and $K_{2,3}$ can be decomposed easily into two graphs $F_{1}$, it follows that $K_{2,3 m}$ has an $F_{1}$-decomposition. Only $K_{2}$ in (2.2) is left non-packed. Hence, the Theorem is proved in this case.

$F_{2}$ : The proof will examine several cases depending on the value of $n$. The following table summarizes the cases $n=5,6,7,8 m$, and $8 m+1$ for $m \geq 1$.

\begin{tabular}{cll}
\hline$n$ & \multicolumn{1}{c}{ packing } & remains for covering \\
\hline 5 & $(0,1,2,3,4) ;(1,3,0,4,2)$ & $(0,2) ;(1,4)$ \\
6 & $(0,1,2,3,4) ;(0,5,4,1,3) ;(0,4,2,5,3)$ & $(0,3),(0,2),(1,5)$ \\
7 & $(0,1,2,3,4) ;(0,2,4,6,1) ;(1,3,5,0,4)$ & \\
& $(1,4,5,6,0) ;(1,5,2,6,3)$ & $(0,3)$ \\
$8 m, 8 m+1$ & $F_{2}-$ decomposition $[4]$ & \\
\hline
\end{tabular}


We still have to prove the theorem for the cases:

$$
n=8 m+k, k=2, \ldots, 7
$$

$\underline{k}=\underline{2}$.

Let

$$
k_{8 m+2}=k_{8 m} \cup k_{2,8 m} \cup k_{2} .
$$

The graph $\mathrm{K}_{8 \mathrm{~m}}$ has an $\mathrm{F}_{2}$-decomposition. since $\mathrm{K}_{2,8 \mathrm{~m}}=2 \mathrm{mK}_{2,4}$ and $\mathrm{K}_{2,4}$ can be decrirposed easily into two graphs $F_{2}$, it follows that $K_{2,8 m}$ has an $F_{2}$-decomposition. Only $K_{2}$ in (2.3) is left non-packed.

$\underline{K}=\underline{3}$.

Let

$$
k_{8 m+3}=K_{8 m+1} \cup K_{2,8 m+1} \cup K_{2}
$$

The craph $K_{8 n+1}$ has an $F_{2}$-deconposition $K_{2,8 m+1}=K_{2,8 m} \cup K_{2,1}$ and $K_{2,8 m 1}$ has an $F_{2}$-decomposition as we saw above. This decomposition of $K_{2,8 m}$ can be done in such a way that the edge $(8 m-1,8 m+2)$ is at one end of the $F_{2}$ which includes it and the point $8 m-1$ is an end-point of that $F_{2}$. Thus we can replace the edge $(8 m-1,8 m+2)$ with the edge $(8 m, 8 m+2)$. Only the edges $(8 m, 8 m+1),(8 m+1,8 m+2)$, $(8 m-1,8 m+2)$ now remain non-packed, and they can be included in one more $F_{2}$.

$\underline{k}=\underline{4}$.

Note that

$$
k_{8 m+4}=K_{8 m} \cup K_{4,8 m} \cup K_{4} .
$$

The graph $K_{8 m}$ hias an $F_{2}$-decomposition. Now

$$
\mathrm{K}_{4,8 \mathrm{~m}} \cup \mathrm{K}_{4}=2(2 \mathrm{~m}-1) \mathrm{K}_{2,4} \cup 2 \mathrm{~K}_{2,4} \cup \mathrm{K}_{4}
$$

and the $2 K_{2,4}$ 's can be selected to be vertex disjoint. Since $K_{2,4}$ has an $F_{2}$-decomposition, so does $2(2 m-1) K_{2,4^{*}}$. We need only to show that $2 K_{2,4} \cup K_{4}$ can be packed by $5 F_{2}$ graphs, leaving two non-packed edges.

Let $V\left(2 K_{c, 4}\right)=\{1,2, \ldots 8, a, b, c, d\}, V\left(K_{4}\right)=\{a, b, c, d\}$.

Then, the 5 graphs of the packing of $2 K_{2,4} \cup k_{4}$ are:

$$
(a, 3, b, c, 8) ;(1, a, d, 7, c) ;(4, b, d, c, 5) ;(a, c, 6, d, 8) ;(1, b, 2, a, 4)
$$

The edges $(d, 5)$ and $(a, b)$ are left non-packed.

$\frac{k=5}{\text { Let }}$.

$$
K_{8 m+5}=K_{8 m+1} \cup K_{4,8 m} \cup K_{4,1} \cup K_{4} .
$$

The graph $K_{8 m+1}$ has an $F_{2}$-decomposition. In the case $K=4$ we saw that $K_{4,8 m} U$ $\mathrm{K}_{4}$ has an $\mathrm{F}_{2}$-packing leaving two non-packed edges.

Let $V\left(k_{4}\right)=\{a, b, c, d\}$ and $V\left(k_{8 m+1}\right)=z_{8 m+1}$. Denote the non-packed edges by $(a, b)$ and $(8 m-1, d)$. We show that $G=K_{4,1} \cup\{(a, b),(8 m-1, d)\}$ has an $F_{2}$-packing leaving two non-packed edges. The $F_{2}$ of this packing is $(8 m-1, d, 8 m, a, b)$. The non-packed edges are: $(c, 8 m)$ and $(8 m, b)$. 
$k=6$.

Write

$$
k_{8 m+6}=k_{8 m} U k_{6,8 m} U k_{6}
$$

The graph $K_{8 m}$ has and $F_{2}$-decomposition. Observe that $K_{6,8 m}=3 K_{2,8 m}$. In the case $k=2$ we saw that $F_{2} \mid K_{2,8 m}$. Tatile 1 shows that $K_{6}$ has $F_{2}$ - packing leaving three non-packed edges as required, and these three can be included in one more $F_{2}$. $\underline{k}=7$.

Let

$$
k_{8 m+7}=k_{8 m+1} \cup k_{6,8 n i} \cup k_{7} .
$$

The graph ${ }^{\prime} 8 m+1$ has an $F_{2}$-decomposition, and $F_{2} \mid K_{6,8 m}$, as was shown above. By Table 1 we know that the graph $k_{7}$ has an $F_{2}$-packing leaving one non-packed edge. The Theorem has now been proved for $F_{2}$ since all cases have been considered. $F_{3}$ : The proof will consider the same cases as the proof for $F_{2}$.

\begin{tabular}{clc}
\hline$n$ & \multicolumn{1}{c}{ packing } & remains for covering \\
\hline 5 & $(0,1,2 ; 3,4) ;(1,4,0 ; 2,3)$ & $(1,3),(3,4)$ \\
6 & $(0,1,2 ; 3,4) ;(3,4,5 ; 0,2) ;(0,3,1 ; 4,5)$ & $(0,2),(0,4),(3,5)$ \\
7 & $(3,2,0 ; 1,6) ;(5,4,1 ; 2,3) ;(1,6,3 ; 4,5)$ & $(2,5)$ \\
& $(2,4,0 ; 3,5) ;(1,5,6 ; 2,4)$ & \\
$8 m, 8 m+1$ & $F_{3}-$ decomposition $[4]$ & \\
\hline
\end{tabular}

\section{Table 2}

lie now have to prove the theorem for the cases:

$$
n=8 m+k, k=2, \ldots, 7, m \geq 1 \text {. }
$$

$k=2$.

Let $K_{8 m+2}$ be as in (2.3). The graph $K_{8 m}$ has an $F_{3}$-decomposition. Since $K_{2,8 m}=$ $2 m K_{2,4}$ and $K_{2,4}$ can be decomposed easily into two $F_{3}$ graphs, it follows that $K_{2,8 n 1}$ has an $F_{3}$-decomposition. Only $K_{2}$ in (2.3) is left non-packed. Hence, the Theorem is proved in this case.

$k=3$.

Let $K_{8 m+3}$ be as in (2.4). $K_{8 m+1}$ has an $F_{3}$-decomposition. $K_{2,8 m+1}=K_{2,8 m} \cup K_{2,1}$. The graph $K_{2,8 m}$ has an $F_{3}$-decomposition as was shown above. Replace the edge $(8 m-4,8 m+2)$ which appears in some $F_{3}$ in the decomposition of $K_{2,8 m}$, with the edge $(8 m, 8 m+2)$. Then the edges $(8 m-4,8 m+2),(8 m+2,8 m+1),(8 m+1,8 m)$ remain non-packed, but could be included in one additional $F_{3}$.

$k=4$.

Let $K_{8 m+4}$ be as in (2.5). The graph $K_{8 m}$ has an $F_{3}$-decomposition. Let, $K_{4,8 m} U$ $K_{4}$ be as in (2.6). Since $K_{2,4}$ has an $F_{3}$-decomposition, so does $2(2 m-1) K_{2,4}$. We show that $2 \mathrm{~K}_{2,4} \cup \mathrm{K}_{4}$ can be packed by five $\mathrm{F}_{3}$ graphs, leaving two non-packed edges. 
Let $V\left(2 K_{2,4}\right)=\{1,2, \ldots 8, a, b, c, d\}$ and $V\left(K_{4}\right)=\{\dot{a}, b, c, d\}$.

Then the five graphs $F_{3}$ are:

$$
(c, 6, d ; 7,8),(d, 5, c ; 7,8),(4, a, c ; d, b),(1, a, b ; 4, d),(a, 3, b ; 1,2) .
$$

The edges $(a, 2)$ and $(a, d)$ are left non-packed.

$\underline{k}=5$.

Let $K_{8 m+5}$ be as in (2.7). The craph $K_{8 m+1}$ has an $F_{3}$-decomposition. In the case $k=i$ we saw that $K_{4,8 m} \cup K_{4}$ has an $F_{3}$-packing leaving two non-packed edges. Let $V^{\prime}\left(K_{f}\right)=\{a, b, c, d\}$ and $V\left(k_{8 m+1}\right)=\bar{L}_{8 m+1}$. Denote the non-packed edges by $(a, d)$ and $(a, \delta,-1)$. The $F_{3}$ graph in $K_{4,1} \cup\{(a, d),,(a, 8 m-1)\}$ is $(b, 8 m, a ; d, 8 m-1)$. The edges $(d, 8 m)$ and $(c, 8 m)$ renia in non-packed.

The froofs for $k=6,7$ are accomplished in the same ways as for $F_{2}$.

Once again all cases have been considered and the proof is complete for $F_{3}$.

$F_{4}$ : it is easy to see that the theorem does not hold for $n=5$ and $n=6$. For $K_{7}$ the graphs $F_{4}$ of the packing are: $(0 ; 1,2,3,4),(1 ; 2,3,4,5),(2 ; 3,4,5,6)$, $(5 ; 4,3,0,6),(6 ; 0,1,3,4)$. The edge $(3,4)$ is left non-packed. Hence, the theorem is proved for $n=7$. For $n=8 m, \varepsilon n+1$ we have an $F_{4}$-deconiposition $[5,6]$. Hence, we again have to prove the theorem for the cases:

$$
n=8 m+k, \quad k=2, \ldots, 7, \quad m \geq 1 .
$$

$k=2$.

Let $k_{8 m+2}=k_{8 m+1} \cup K_{1,8 m+1}$. The graph $K_{8 m+1}$ has an $F_{4}$-decomposition. $K_{1,8 m+1}$ is a star that can easily be packed by $2 \mathrm{~m}$ stars $F_{4}$, leaving one non-packed edged.

$k=3$.

Let $K_{8 m+?}=K_{8 m} \cup K_{3,8 m} \cup K_{3}$. The graph $K_{8 m}$ has an $F_{4}$-decomposition. Let $K_{3,8 m}=$ $3 K_{1,8 m}$. Since the graph $K_{1,8 m}$ can be decomposed into $2 m$ stars $F_{4}$, it follows that $K_{3,8 m}$ also has an $F_{4}$-decomposition. Let $V\left(K_{3}\right)=\{a, b, c\}$, and create a decomposition of $K_{3,8 m}$ which includes the three stars $(a ; x, y, z, u),(b ; x, y, z, u)$, $(c ; x, y, z, u)$. Replace the edge $(a, u)$ by $(a, b)$, the edge $(b, u)$ by $(b, c)$, and the edge $(c, u)$ by $(c, a)$. We did not spoil any star of the decomposition of $k_{3,8 m}$ and the star $(u ; a, b, c)$ of three branches is left non-packed.

$k=4$.

Let $K_{8 m+4}=K_{8 m} \cup K_{4,8 m} \cup K_{4}$. The graph $K_{8 m}$ has an $F_{4}$-decomposition. Let $K_{4,8 m}=4 K_{1,8 m}$. The graph $K_{1,8 m}$ can be decomposed into $2 m$ stars $F_{4}$ so $K_{4,8 m}$ has an $F_{4}$-decomposition. Let $V\left(K_{4}\right)=\{a, b, c, d\}$, and consider the subgraph $K_{4,4}$ of $K_{4,8 m}$ whose vertices are given by $V\left(K_{4,4}\right)=\{a, b, c, d\} \cup\{8 m-1,8 m-2,8 m-3,8 m-4\}$. The $\mathrm{F}_{4}$ decomposition of $K_{4,8 m}$ can be arranged in such a way that our $K_{4,4}$ is made up of the four $F_{4}$ graphs $(a ; 8 m-1,8 m-2,8 m-3,8 m-4),(b ; 8 m-1,8 m-2,8 m-3,8 m-4)$, $(c ; 8 m-1,8 m-2,8 m-3,8 m-4)$ and $(d ; 8 m-1,8 m-2,8 m-3,8 m-4)$. Replace the edges $(a, 8 m-1)$, $(b, 8 m-1),(c, 8 m-1),(d, 8 m-1)$ with the edges $(a, c),(a, b),(b, c),(c, d)$, respectively we now have a new $F_{4}$ graph, nariely $(8 m-1 ; a, b, c, d)$. The edges $(a, d)$ and $(b, d)$ are the only one which remain non-packed. 
$\underline{k}=5$.

Let $K_{8 m+5}=K_{8 m} \cup K_{5,8 m} \cup K_{5}$. As before $K_{8 m}$ and $K_{5,8 m}$ have $F_{4}$-decompositions. Now $K_{5}=K_{4} \cup K_{1,4}$ so we can complete the proof in the same way as in the case $k=$ 4.

$\underline{k}=6$.

Let $K_{8 m+6}=K_{8 m} \cup K_{6,8 m} \cup K_{6}$. The graphs $K_{8 m}$ and $K_{6,8 m}$ have $F_{4}$-decompositions. Let $V\left(K_{6}\right)=\left\{v_{1}, v_{2}, v_{3}, v_{4}, v_{5}, v_{6}\right\}$. Graph $K_{6}$ can be packed with the two $F_{4}$ $\left\{v_{1} ; v_{2}, v_{3}, v_{4}, v_{5}\right\}$ and $\left\{v_{2} ; v_{3}, v_{4}, v_{5}, v_{6}\right\}$. The induced graph on $\left\{v_{3}, v_{4}, v_{5}, v_{6}\right\}$ is $k_{4}$. Hence, we can complete the proof here as in the case $k=4$, leaving the edges $\left(v_{5}, v_{6}\right),\left(v_{4}, v_{6}\right)$ non-packed. Those edges together with the non-packed edge $\left(v_{1}, v_{6}\right)$ accomplish the proof of the theorem in this case.

$k=7$.

Let $\mathrm{K}_{8 \mathrm{~m}+7}=\mathrm{K}_{8 \mathrm{~m}} \cup \mathrm{K}_{7,8 \mathrm{~m}} \cup \mathrm{K}_{7}$. The graphs $\mathrm{K}_{8 \mathrm{~m}}$ and $\mathrm{K}_{7,8 \mathrm{~m}}$ have $\mathrm{F}_{4}$-decompositions ard we apply the $F_{4}$-packing shown for $K_{7}$ at the beginning of this case.

This completes the proof of the theorem for $F_{4}$.

\section{REFERENCES}

1. BCINEKE, L. W. A Survey of Packing and Covering of Graphs. The Many Faces of Graph Theory. Ed G. Chartrand and S. F. Kapoor. Berlin, Heidelberg, New York 1969, p. 45.

2. RODITTY, Y. Packing and Covering of the Complete Graph with a Graph G of Four Vertices or Less. J. Combin. Theory Ser. A, 34 (1983) No. 2, 231-243.

3. BERMOND, J. C., hUANG, C., ROSA, A. and SOTTEAU, D. Decomposition of Complete Graphs into Isomorphic Subgraphs with Five Vertices, ARS Combin., 10 (1980) p. 211-254

4. RAY-CHAUDHURI, D. K., and WILSON, R. M. The Existence of Resolvable Designs, $A$ Survey of Combinatorial Theory, (Ed. J. N. Srivastava et al.), North-Holland, Amsterdam 1973, p. 361-376.

5. TARSI, M. Decomposition of Complete Multigraphs into Stars, Discrete Math. $\underline{26}$ (1979) p. 273-278.

6. Yamomcto, Sumiyasu et al. On Claw-Decomposition of Complete Graphs and Complete Digraphs. Hiroshima Math. J. $\underline{5}$ (1975) 33-42. 


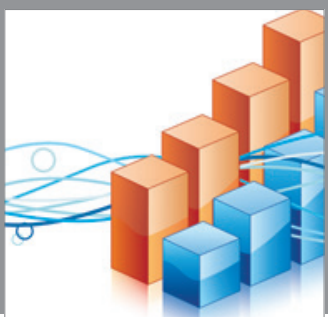

Advances in

Operations Research

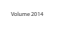

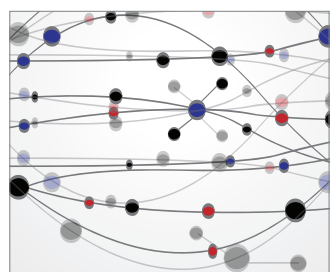

\section{The Scientific} World Journal
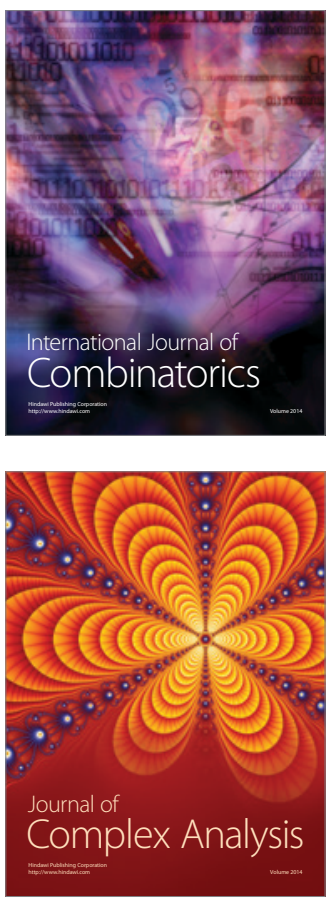

International Journal of

Mathematics and

Mathematical

Sciences
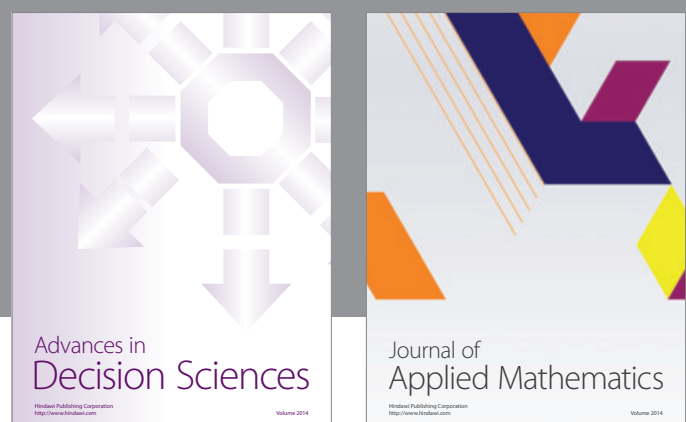

Journal of

Applied Mathematics
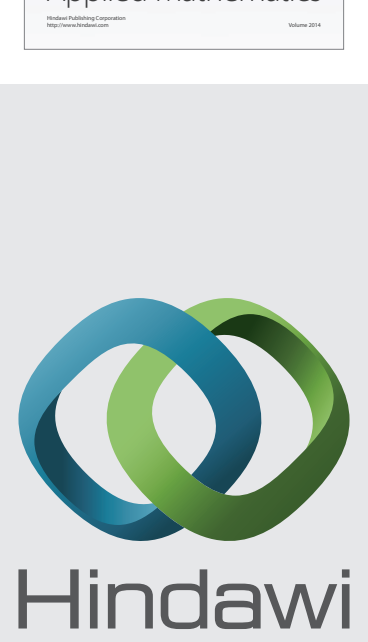

Submit your manuscripts at http://www.hindawi.com
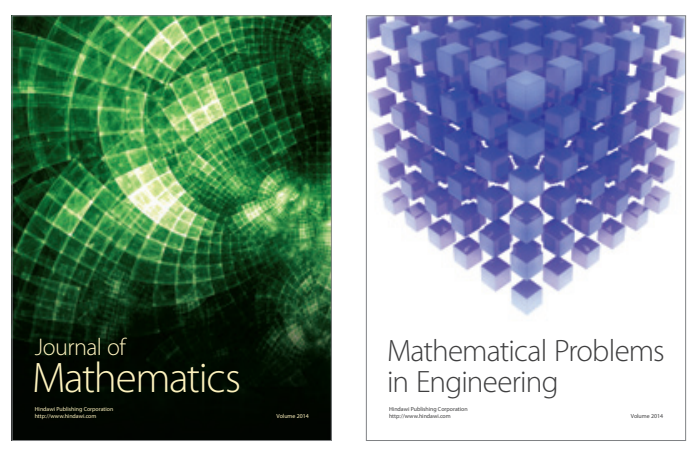

Mathematical Problems in Engineering
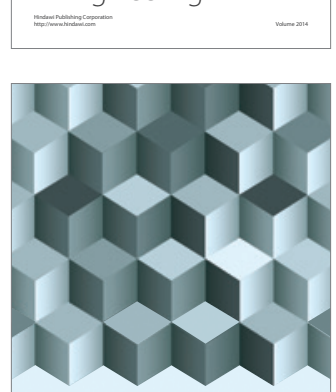

Journal of

Function Spaces
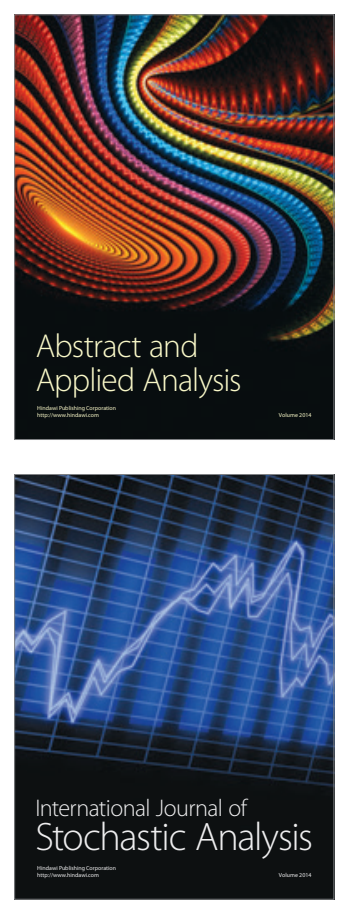

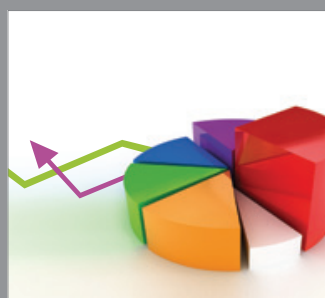

ournal of

Probability and Statistics

Promensencen
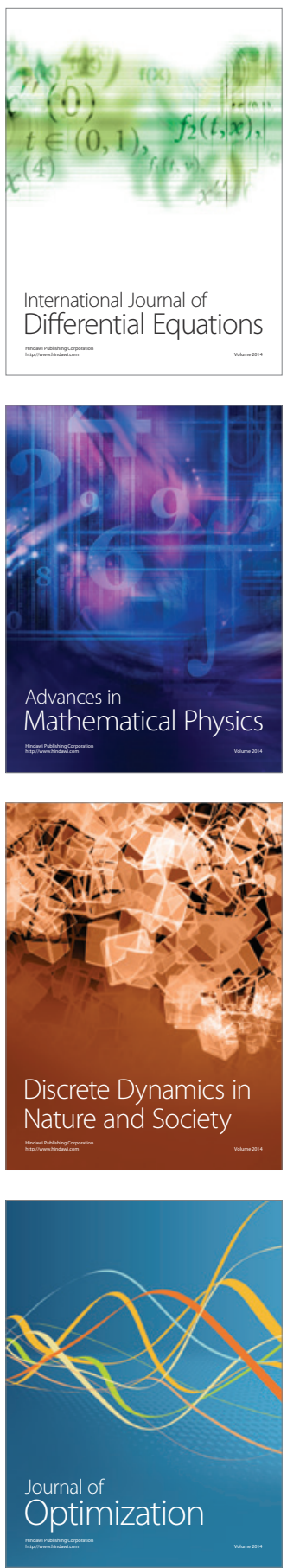